\title{
Correlation Between Psoriasis Severity and Nonalcoholic Fatty Liver Disease Degree Measured Using Controlled Attenuation Parameter
}

This article was published in the following Dove Press journal:

Psoriasis: Targets and Therapy

\author{
Nico Gandha (iD) \\ Larisa Paramitha Wibawa (iD) ${ }^{\prime}$ \\ Tjut Nurul Alam Jacoeb' \\ Andri Sanityoso Sulaiman ${ }^{2}$ \\ 'Department of Dermatology and \\ Venereology, Faculty of Medicine \\ Universitas Indonesia/Cipto \\ Mangunkusumo Hospital, Jakarta 10430, \\ Indonesia; ${ }^{2}$ Department of Internal \\ Medicine, Faculty of Medicine Universitas \\ Indonesia/Cipto Mangunkusumo Hospital, \\ Jakarta 10430, Indonesia
}

Background: A growing body of evidence links psoriasis to several metabolic disorders, but the causal relationship between psoriasis and nonalcoholic fatty liver disease (NAFLD) remains understudied.

Purpose: To measure the correlation between the severity of psoriasis and the degree of NAFLD. Patients and Methods: A cross-sectional study was conducted on adult patients with psoriasis in the Dermatovenereology Outpatient Clinic of Cipto Mangunkusumo Hospital from December 2017 through February 2018. Psoriasis severity (psoriasis area and severity index [PASI] and body surface area [BSA]) was recorded and compared with NAFLD degree measured by controlled attenuation parameter (CAP).

Results: A total of 36 subjects were enrolled with an average age of 49.08 years $( \pm 15.52$ years). The proportions of mild, moderate, and severe psoriasis were $50 \%, 27.8 \%$, and $22.2 \%$, respectively. Median of PASI was $6.1(2-38.4)$ and BSA was 7.5 (2-93). The proportion of NAFLD was $77.8 \%$. The mean of the CAP score was $250.03 \pm 45.64$. There was no statistically significant correlation between psoriasis severity based on PASI and CAP score $(r=0.258 ; p=0.128)$. However, if the degree of psoriasis was based on BSA, a significant correlation was found $(\mathrm{r}=0.382 ; \mathrm{p}=0.021)$. The body mass index (BMI) and abdominal circumference were significantly correlated with CAP score $(r=0.448, p=$ 0.006 and $\mathrm{r}=0.485, \mathrm{p}=0.003$, respectively).

Conclusion: Psoriasis extension correlates with NAFLD severity; further studies should assess in detail the effect of therapies on this pathophysiological link.

Keywords: psoriasis, nonalcoholic fatty liver disease, severity, PASI score, controlled attenuation parameter

\section{Introduction}

Psoriasis is a chronic skin inflammatory disorder with genetic predisposition with prevalence ranges from $0.1 \%$ to $11.8 \% .^{1,2}$ Numerous evidences have demonstrated that the inflammatory process in psoriasis is not limited to the skin, but also involves systemic response. ${ }^{3-5}$ A number of diseases have been associated with psoriasis including, among others, nonalcoholic fatty liver disease (NAFLD). ${ }^{6}$ NAFLD is a spectrum of hepatic disorders ranging from a relatively benign fatty liver (nonalcoholic fatty liver) to an inflammatory fatty liver (nonalcoholic steatohepatitis; NASH) that can progress into fibrosis, cirrhosis, or even hepatocellular
Wibawa

Department of Dermatology and Venereology, Faculty of Medicine

Universitas Indonesia/Cipto

Mangunkusumo Hospital, Jl. Diponegoro

No. 7I, Jakarta 10430, Indonesia

Tel +6221 31935383

Email larisa.paramitha@ui.ac.id
Psoriasis: Targets and Therapy 2020:10 39-44 
Table I Clinical Characteristics of Patients with Psoriasis at Cipto Mangunkusumo Hospital, Jakarta $(n=36)$

\begin{tabular}{|c|c|c|c|c|c|}
\hline Characteristics & Number & Percentage & $\begin{array}{l}\text { Mean/ } \\
\text { Median }\end{array}$ & Standard Deviation & Minimum- Maximum \\
\hline $\begin{aligned} \text { PASI } & \\
\text { - } & \text { Mild } \\
\text { - } & \text { Moderate } \\
\text { - } & \text { Severe }\end{aligned}$ & $\begin{array}{l}18 \\
10 \\
8\end{array}$ & $\begin{array}{l}50 \\
27.8 \\
22.2\end{array}$ & 6.1 & & $2-38.4$ \\
\hline 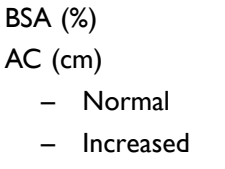 & $\begin{array}{l}10 \\
26\end{array}$ & $\begin{array}{l}27.8 \\
72.2\end{array}$ & $\begin{array}{l}7.5 \\
89\end{array}$ & \pm 10.23 & $2-93$ \\
\hline $\begin{aligned} \text { BMI }\left(\mathrm{kg} / \mathrm{m}^{2}\right) \\
-\quad \text { Normal } \\
-\quad \text { Obese }\end{aligned}$ & $\begin{array}{l}16 \\
20\end{array}$ & $\begin{array}{l}44.4 \\
55.6\end{array}$ & 26.36 & \pm 3.95 & \\
\hline $\begin{aligned} \text { CAP }(\mathrm{dB} / \mathrm{m}) \\
-\quad \text { No NAFLD } \\
-\quad \text { NAFLD }\end{aligned}$ & $\begin{array}{l}8 \\
28\end{array}$ & $\begin{array}{l}22.2 \\
77.8\end{array}$ & 250.03 & \pm 45.64 & \\
\hline
\end{tabular}

Abbreviations: AC, abdominal circumference; BSA, body surface area; CAP, controlled attenuation parameter; BMI, body mass index; PASI, psoriasis area and severity index; NAFLD, nonalcoholic fatty liver disease.

carcinoma. NAFLD is linked to metabolic syndrome and thought to be a hepatic manifestation of metabolic syndrome. ${ }^{7,8}$ Knowledge of the association between psoriasis and NAFLD is necessary in order to early detect and provide immediate treatment before further complication occurs.

Earlier studies conducted in Italy and India reported that NAFLD was more common among patients with psoriasis compared to non-psoriatic controls ( $47 \%$ vs $28 \%$ and $17.4 \%$

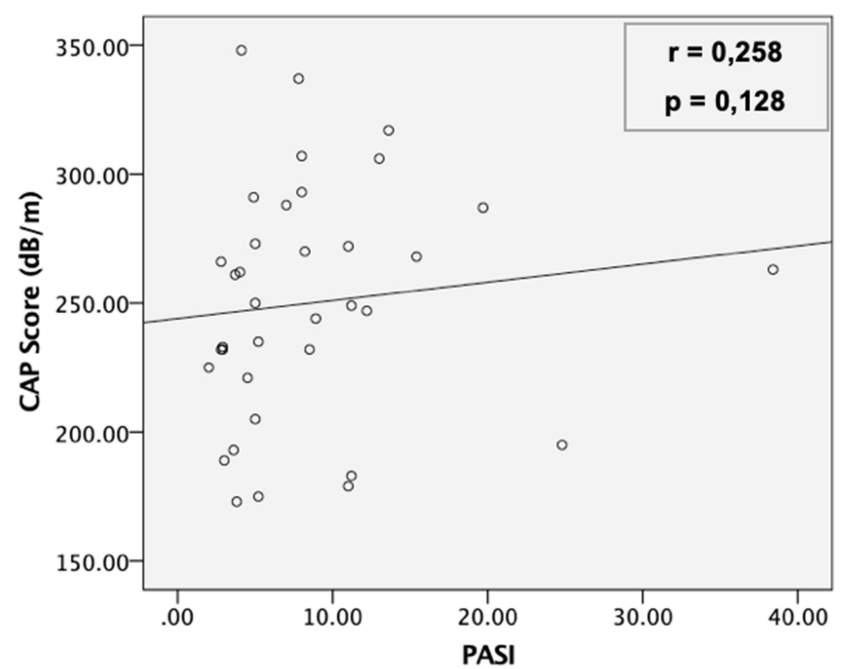

Figure I Correlation between PASI and CAP score among patients with psoriasis at Cipto Mangunkusumo Hospital, Jakarta $(n=36)$.

Abbreviations: CAP, controlled attenuation parameter; PASI, psoriasis area and severity index. vs $7.9 \%$, respectively). ${ }^{9}$ These studies used ultrasound to confirm the diagnosis of NAFLD. Currently, a novel imaging modality to measure the degree of fatty liver is available, namely controlled attenuation parameter (CAP) by employing transient elastography (fibroscan ${ }^{\circledR}$ ). CAP is a rapid, operator-independent, and less invasive diagnostic tool possessing higher sensitivity and specificity compared with ultrasound. ${ }^{10}$ To the best of our knowledge, there is no study conducted in Indonesia that aims to investigate the association between psoriasis and NAFLD. This is also the first study in the world that aims to provide information regarding the correlation between psoriasis severity and NAFLD degree measured using CAP.

\section{Patients and Methods}

This study was conducted using cross-sectional design and involved patients with psoriasis vulgaris in Cipto Mangunkusumo Hospital, Jakarta. This tertiary hospital serves as the national referral center in Indonesia. Clinical history taking and a physical examination were conducted at the dermatology and venereology outpatient clinic. CAP assessment for determining the degree of fatty liver was conducted in a procedure room of the hepatology clinic. The study was conducted from December 2017 to February 2018.

\section{Study Population and Subjects}

The inclusion criteria includes adult patients with psoriasis vulgaris who agreed to participate in this study and 


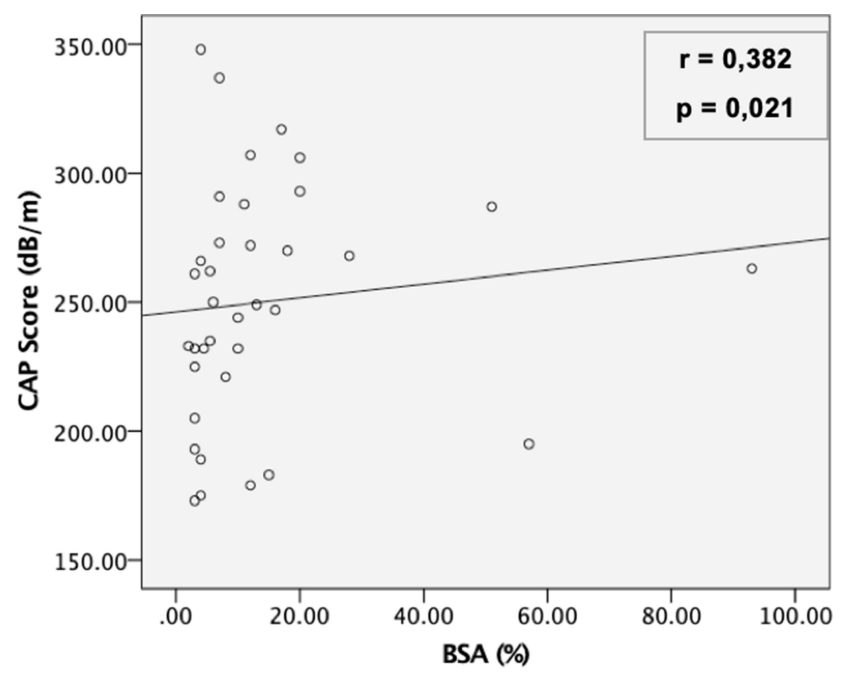

Figure 2 Correlation between BSA and CAP score among patients with psoriasis at Cipto Mangunkusumo Hospital, Jakarta $(n=36)$.

Abbreviations: BSA, body surface area; NAFLD, nonalcoholic fatty liver disease.

provide written informed consent. The exclusion criteria consists of patients with a secondary cause of fatty liver, including hepatitis $\mathrm{B}$ and $\mathrm{C}$ infection (confirmed by the patient's clinical history or laboratory results obtained from medical record), history of alcohol consumption at least once in a week for the past year, treatment with systemic medications that can induce fatty liver (amiodarone, methotrexate, tamoxiphen, corticosteroid, valproic acid, antiretroviral drugs) for the past 4 weeks, and pregnancy. The sample size was calculated using a sample size formula for correlative studies with the minimum number of samples needed to show significant difference being 33 subjects. ${ }^{11}$

\section{Recording of Baseline Characteristics}

Data regarding patients' characteristics, body mass index (BMI), and abdominal circumference, were recorded. BMI was calculated according to a classification system proposed by Asia-Pacific Task Force. Obesity was defined as BMI above $25 \mathrm{~kg} / \mathrm{m}^{2}$. Central obesity was defined as abdominal circumference above 90 centimeters in men and 80 centimeters in women. ${ }^{12}$

\section{Assessment of Psoriasis Severity}

Assessment of the degree of psoriasis severity was done using psoriasis area and severity index (PASI) as described by Fredriksson and Petterson. PASI score of $<7$ was considered as mild psoriasis, $7-12$ as moderate, and $>12$ as severe. ${ }^{13}$ In addition, the body surface area (BSA) score was recorded and calculated based on the patient's palm size considered as $1 \%$ of the total body surface area. For those who received systemic therapy (acitretin, cyclosporine) or phototherapy for psoriasis, the medical records were evaluated to find their pre-treatment PASI and BSA scores.

\section{Controlled Attenuation Parameter Procedure}

CAP was conducted using transient elastography (fibroscan ${ }^{\circledR}$, Echosens, Paris, France) according to the standard protocol to assess the grade of fatty liver. All patients were scanned using the $\mathrm{M}$ probe. When indicated by the equipment, patients were rescanned using the XL probe. CAP was conducted until at least 10 accurate shots were made, with minimum success ratio (number of accurate shots divided by number of shots in total) of $60 \%$. The interquartile range (IQR)/median must be less than $30 \%$ and IQR of CAP less than $40 \mathrm{~dB} / \mathrm{m}^{14}$ This measure was considered failed if no accurate shot was made after 10 attempts. Final CAP score was the average of all CAP results and was recorded in $\mathrm{dB} / \mathrm{m}$. A CAP score of $>215$ $\mathrm{dB} / \mathrm{m}$ indicated the presence of fatty liver. ${ }^{10}$ Diagnosis of NAFLD was made by confirming the presence of fatty liver and excluding other possible causes of secondary fatty liver such as hepatitis, high alcohol intake, and certain fatty liver-inducing medications.

\section{Data Processing and Statistical Analysis}

The data was analyzed using Statistical Program for Social Science (SPSS) software version 23. Analysis of distribution was conducted using Kolmogorov-Smirnov test with $\mathrm{p}$ value of $>0.05$ recorded as normal distribution. Normally-distributed data was presented in mean and standard deviation. Correlative analysis between two continuous variables was conducted using Pearson correlation coefficient (for data with normal distribution) or Spearman correlation (for data with abnormal distribution). Correlation between the two variables was expressed in $r$ value. The correlation was considered strong if the $r$ value $=0.7-1.0$, moderate if the $r$ value $=0.3-0.699$, and weak if the $r$ value $=0-0.299$. Independent variable evaluated in this study was the severity of psoriasis. Whilst, the dependent variable was NAFLD degree. The controlled variables are as follows: alcohol intake, hepatitis $\mathrm{B}$ and $\mathrm{C}$, medications that induce fatty liver formation, and pregnancy. 


\section{Code of Ethics}

The study was approved by the Health Research Ethics Committee of the Faculty of Medicine, Universitas Indonesia (1007/UN2.F1/ETIK/2017) and granted research permission from the Head of Research Division of Cipto Mangunkusumo Hospital (LB.02.01/X.2/99/ 2017). The study was conducted in accordance with the Declaration of Helsinki and all patients provided their written informed consent.

\section{Results}

During the study period, a total of 36 subjects were involved in the analysis. The proportion of female participants was slightly higher in this study population $(55.6 \%)$. The mean age was $49.08 \pm 15.52$ years. In this study, most of the participants $(66.7 \%)$ suffered from type I psoriasis (age of onset before 40 years). Mean of disease duration among the study population was $12.46 \pm 9.26$ years. There were 6 patients on phototherapy and 3 patients on systemic therapy (cyclosporine in 2 patients and sulfasalazine in 1 patient). Among all patients, 27 measured with $M$ probe CAP. No patient was deemed to have failed the CAP measurement.

The descriptive analysis on patients' clinical characteristics based on the severity of psoriasis, abdominal circumference, BMI, and the presence of NAFLD are shown in Table 1.

Weak correlation was found between the severity of psoriasis based on PASI and NAFLD degree based on CAP score, but not statistically significant (Figure 1). A significant correlation was found between the severity of psoriasis based on BSA and NAFLD degree based on CAP score. The correlation was positive with moderate strength (Figure 2).

\section{Discussion}

The primary objective of this study was to conduct a correlative analysis between the severity of psoriasis and the degree of NAFLD. A 2014 systematic review of seven case-control studies reported that patients with psoriasis had a twofold greater risk of developing NAFLD compared to non-psoriasis controls. It was also reported that the risk was greater in patients who suffer from moderate-severe psoriasis. In those studies, the severity of psoriasis was assessed using PASI and diagnosis of NAFLD was confirmed by liver ultrasound. ${ }^{15}$
The mechanisms underlying the association between the severity of psoriasis and NAFLD are thought to involve chronic mild systemic inflammation and insulin resistance. Secretion of pro-inflammatory cytokines and growth factors within cutaneous psoriatic lesions produce systemic effects that induce insulin resistance and subsequent metabolic changes and development of fatty liver. Several cytokines, including tumor necrosis factor alpha (TNF- $\alpha$ ), interleukin (IL) 1, 2, 6, and 17, are known to influence glucose metabolism and insulin sensitivity in hepatocytes and adipocytes leading to uncontrolled lipolysis and increased hepatic free fatty acid deposition. ${ }^{15}$ Conversely, NAFLD can affect psoriasis severity through the release of inflammatory mediators from the hepatocyte, namely reactive oxygen species (ROS), C-reactive protein (CRP), and IL-6. ${ }^{9}$

In our study, the correlative analysis between PASIbased psoriasis severity and CAP-based NAFLD degree shows a weak positive correlation and no statistically significant result (Figure 1). To the best of our knowledge, no study has investigated the correlation between psoriasis severity and NAFLD degree using CAP, so this study is the first in the world.

Utilization of CAP is the strength of our study. CAP is rapid, operator-independent, less invasive, and reliable. A study conducted in 2012 reported that the sensitivity and specificity of CAP were higher than ultrasound. ${ }^{10}$ CAP can detect early development of fatty liver affecting $5-10 \%$ of hepatocytes whereas ultrasound can only detect if fatty liver affects at least $30 \%$ of hepatocytes. A study in 2014 reported that the CAP score was positively correlated with the percent of fatty liver found on liver biopsy, a gold standard diagnostic modality for fatty liver. ${ }^{16}$

Correlation between psoriasis severity and NAFLD degree is significant if using BSA in assessing psoriasis severity instead of PASI. Research on the correlation between BSA and NAFLD has never been conducted before. Only a small number of studies reporting the correlation between BSA and systemic inflammatory disorders are found. A retrospective study in the United States in 2010 reported that the BSA-based psoriasis severity was positively correlated with the risk of coronary heart disease occurring in the following 10 years. ${ }^{17}$

BSA describes the extent of psoriatic lesions. Even though BSA provides less detailed assessment as compared to PASI, BSA has potential use for measuring the severity of psoriasis in terms of the extent of inflamed area. Inflammation in psoriasis involves not only local 
areas of the skin, but also systemic responses, and this underlies the link between psoriasis and comorbidities including coronary heart disease and NAFLD., ${ }^{9,18}$ The result of this study suggests that the extent of psoriasis skin lesions may have more preponderant effect on the degree of NAFLD. So that when calculated together with the scaling and infiltration components in PASI, the relationship becomes insignificant.

However, there is still an indirect correlation between PASI and CAP score in our study. A German study in 2008 reported that the BSA score had a strong positive correlation with PASI $(\mathrm{r}=0.81 ; \mathrm{p} \leq 0.001) .{ }^{19}$ Similarly, our study also finds a strong positive correlation between BSA and PASI ( $r$ $=0.91 ; \mathrm{p}<0.0001)$. The indirect correlation between PASI and CAP score is bridged by the BSA component of PASI.

There are other possible explanations why our study found the degree of NAFLD correlated with BSA, but not with PASI. This could be due to the fact that the PASI and BSA data of patients receiving either systemic or phototherapy were obtained retrospectively from medical records. A systematic review in 2010 showed that PASI had better interobserver reliability than $\mathrm{BSA}^{20}$ But a recent study in 2018 reported that BSA was the PASI component possessing the best interobserver reliability, while scaling and infiltration components had lower interobserver reliability. ${ }^{21}$ So, BSA may be more reliable to be used as a variable in the analysis and provides the significant correlation result with CAP.

The reason we used the PASI and BSA data from medical records was because of the chronic characteristics of fatty liver. A study in 2012 reported patients with psoriasis who received anti-TNF- $\alpha$ (etanercept) and PUVA therapies for 6 months, with a significant decrease in PASI score but no significant reduction in the degree of fatty liver measured by ultrasound. ${ }^{22}$ Therefore, for the patients undergoing either systemic or phototherapy, we recorded the last PASI and BSA scores before they received that treatment.

Apart from those mentioned above, the insignificant correlation between PASI and the degree of NAFLD in this study could be due to other factors that influence the degree of NAFLD, such as obesity and central obesity. Patients who suffer from psoriasis and NAFLD have a risk of increasing BMI and abdominal circumference. ${ }^{10,18,23}$ In this study, it was found that BMI and abdominal circumference had a significant correlation with the degree of NAFLD. Both BMI and abdominal circumference were found to have a positive correlation with moderate correlation strength with the degree of NAFLD based on CAP score $(\mathrm{r}=$ $0.448, p=0.006$ and $r=0.485, p=0.003$, respectively).

This results are in accordance with the results of previous studies. A 2015 Iranian study reported that psoriasis patients with NAFLD as a comorbidity had higher mean BMI and abdominal circumference compared to those without NAFLD. ${ }^{18}$ A 2017 Portuguese study reported that BMI was positively correlated with the degree of fatty liver measured using CAP and the correlation was moderate $(\mathrm{r}=0.533 ; \mathrm{p}<0.001) .^{23}$ Meanwhile, a 2012 French study reported that abdominal circumference was moderately positively correlated with the CAP score $(\mathrm{r}=$ 0.357; $\mathrm{p}<0.0001) .{ }^{10}$ However, NAFLD was assessed using ultrasound in the Iranian study, and the subjects in Portugal and France were patients with chronic liver disease. There has been no previous study using CAP to measure fatty liver involving patients with psoriasis.

The association of psoriasis, NAFLD, obesity, and central obesity is thought to be mediated by inflammatory pathways and deposition of adipocytes. Abdominal circumference is correlated with visceral adipose tissue and associated with both peripheral and hepatic insulin resistance that induce the development of fatty liver. ${ }^{24}$ Activated macrophages in adipose tissue induce adipocytes to produce inflammatory mediators that play a major role in maintaining the chronic inflammatory state in obesity and underlie the relationship among obesity, psoriasis, and NAFLD. A decrease in adiponectin and an increase in TNF- $\alpha$ level are found in patients with psoriasis, NAFLD, and obesity. ${ }^{12}$

The limitation of the study is that there is no laboratory data to strengthen the causal relationship between the related variables, eg inflammatory and metabolic markers associated with metabolic syndrome and insulin resistance.

\section{Conclusion}

There is a significant correlation between the severity of psoriasis assessed using BSA and the degree of NAFLD based on CAP. Screening and evaluation of NAFLD is required, particularly in patients with extensive skin lesions, as well as those with a high BMI and abdominal circumference. Further studies are needed to explore a more detailed pathophysiological link, including the effect of therapies, between psoriasis and NAFLD.

\section{Acknowledgments}

The authors thank Rino Alvani Gani (Head of Hepatology Division, Department of Internal Medicine, Faculty of 
Medicine Universitas Indonesia) for the permission and support of this collaboration research.

\section{Disclosure}

The authors report no conflicts of interest in this work.

\section{References}

1. Michalek IM, Loring B, John SM. A systematic review of worldwide epidemiology of psoriasis. J Eur Acad Dermatol Venereol. 2017;31 (2):205-212. doi: $10.1111 / j d v .13854$

2. Parisi R, Symmons DP, Griffiths CE, Ashcroft DM. Global epidemiology of psoriasis: a systematic review of incidence and prevalence. J Invest Dermatol. 2013;133(2):377-385. doi:10.1038/ jid.2012.339

3. Kridin K, Ludwig RJ, Damiani G, Cohen AD. Increased risk of pemphigus among patients with psoriasis: a large-scale cohort study. Acta Derm Venereol. 2020. doi:10.2340/00015555-3607

4. Kridin K, Vanetik S, Damiani G, Cohen AD. Big data highlights the association between psoriasis and fibromyalgia: a population-based study. Immunol Res. 2020;68(3):135-140. doi:10.1007/s12026-02009135-7

5. Watad A, Bragazzi NL, McGonagle D, et al. Systemic sclerosis is linked to psoriasis and may impact on patients' survival: a large cohort study. J Clin Med. 2019;8(4):521. doi:10.3390/jcm8040521

6. Farley E, Menter A. Psoriasis: comorbidities and associations. G Ital Dermatol Venereol. 2011;146(1):9-15.

7. de Alwis NM, Day CP. Non-alcoholic fatty liver disease: the mist gradually clears. $J$ Hepatol. 2008;48:S104-12. doi:10.1016/j. jhep.2008.01.009

8. Hasan I, Gani RA, Machmud R. Prevalence and risk factors for nonalcoholic fatty liver in Indonesia. $J$ Gastroenterol Hepatol. 2002; 17:S154

9. Gisondi P, Targher G, Zoppini G, Girolomoni G. Non-alcoholic fatty liver disease in patients with chronic plaque psoriasis. Hepatology. 2009;51(4):758. doi:10.1016/j.jhep.2009.04.020

10. de Ledinghen V, Vergniol J, Foucher J, Merrouche W, le Bail B. Noninvasive diagnosis of liver steatosis using controlled attenuation parameter (CAP) and transient elastography. Liver Int. 2012;32 (6):911-918. doi:10.1111/j.1478-3231.2012.02820.x

11. Hulley SB, Cummings SR, Browner WS, Grady D, Newman TB. Designing Clinical Research: An Epidemiologic Approach. 4th ed. Philadelphia, PA: Lippincott Williams \& Wilkins; 2013:Appendix $6 \mathrm{C}$, page 79 .

12. Armstrong AW, Harskamp CT, Armstrong EJ. The association between psoriasis and obesity: a systematic review and meta-analysis of observational studies. Nutr Diabetes. 2012;2:e54. doi:10.1038/nutd.2012.26
13. Schmitt J, Wozel G. The psoriasis area and severity index is the adequate criterion to define severity in chronic plaque-type psoriasis. Dermatology. 2005;210(3):194-199. doi:10.1159/ 000083509

14. Wong VW, Petta S, Hiriart JB, et al. Validity criteria for the diagnosis of fatty liver by $\mathrm{M}$ probe-based controlled attenuation parameter. J Hepatol. 2017;67(3):577-584. doi:10.1016/j.jhep.2017.05.005

15. Candia R, Ruiz A, Torres-Robles R, Chavez-Tapia N, MendezSanchez N, Arrese M. Risk of non-alcoholic fatty liver disease in patients with psoriasis: a systematic review and meta-analysis. $J$ Eur Acad Dermatol Venereol. 2015;29(4):656-662. doi:10.1111/ jdv. 12847

16. de Ledinghen V, Vergniol J, Capdepont M, et al. Controlled attenuation parameter (CAP) for the diagnosis of steatosis: a prospective study of 5323 examinations. J Hepatol. 2014;60(5):1026-1031.

17. Kamalpour L, Perry A, Leslie H, Hill N. Body surface area as a measure of psoriasis-associated cardiovascular disease risk. $J \mathrm{Am}$ Acad Dermatol. 2010;62(3):AB68.

18. Abedini R, Salehi M, Lajevardi V, Beygi S. Patients with psoriasis are at a higher risk of developing nonalcoholic fatty liver disease. Clin Exp Dermatol. 2015;40(7):722-727. doi:10.1111/ced.12672

19. Henseler T, Schmitt-Rau K. A comparison between BSA, PASI, PLASI and SAPASI as measures of disease severity and improvement by therapy in patients with psoriasis. Int J Dermatol. 2008;47 (10):1019-1023. doi:10.1111/j.1365-4632.2008.03753.x

20. Puzenat E, Bronsard V, Prey S, et al. What are the best outcome measures for assessing plaque psoriasis severity? A systematic review of the literature. J Eur Acad Dermatol Venereol. 2010;24 (Suppl 2):10-16.

21. Fink C, Alt C, Uhlmann L, Klose C, Enk A, Haenssle HA. Intra- and interobserver variability of image-based PASI assessments in 120 patients suffering from plaque-type psoriasis. J Eur Acad Dermatol Venereol. 2018;32(8):1314-1319. doi:10.1111/jdv.14960

22. Campanati A, Ganzetti G, Di Sario A, et al. The effect of etanercept on hepatic fibrosis risk in patients with non-alcoholic fatty liver disease, metabolic syndrome, and psoriasis. $J$ Gastroenterol. 2013;48(7):839-846. doi:10.1007/s00535-012-0678-9

23. Andrade P, Rodrigues S, Rodrigues-Pinto E, et al. Diagnostic accuracy of controlled attenuation parameter for detecting hepatic steatosis in patients with chronic liver disease. GE Port J Gastroenterol. 2017;24(4):161-168. doi:10.1159/000453364

24. Pan JJ, Fallon MB. Gender and racial differences in nonalcoholic fatty liver disease. World J Hepatol. 2014;6(5):274-283. doi:10.4254/ wjh.v6.i5.274
Psoriasis: Targets and Therapy

\section{Publish your work in this journal}

Psoriasis: Targets and Therapy is international, peer-reviewed, open access journal focusing on psoriasis, nail psoriasis, psoriatic arthritis and related conditions, identification of therapeutic targets and the optimal use of integrated treatment interventions to achieve improved outcomes and quality of life. Visit http://www.dovepress. com/testimonials.php to read real quotes from published authors. 